\title{
Correlation between lung infection severity and clinical laboratory indicators in patients with COVID-19: a cross-sectional study based on machine learning
}

Xingrui Wang ${ }^{1,2+}$, Qinglin Che ${ }^{3+}$, Xiaoxiao $\mathrm{Ji}^{1}$, Xinyi Meng ${ }^{1}$, Lang Zhang ${ }^{1}$, Rongrong Jia ${ }^{1}$, Hairong Lyu ${ }^{1}$, Weixian Bai ${ }^{1}$, Lingjie $\operatorname{Tan}^{1}$ and Yanjun Gao ${ }^{1,2^{*}}$

\begin{abstract}
Background: Coronavirus disease 2019 (COVID-19) has caused a global pandemic that has raised worldwide concern. This study aims to investigate the correlation between the extent of lung infection and relevant clinical laboratory testing indicators in COVID-19 and to analyse its underlying mechanism.

Methods: Chest high-resolution computer tomography (CT) images and laboratory examination data of 31 patients with COVID-19 were extracted, and the lesion areas in CT images were quantitatively segmented and calculated using a deep learning (DL) system. A cross-sectional study method was carried out to explore the differences among the proportions of lung lobe infection and to correlate the percentage of infection (POI) of the whole lung in all patients with clinical laboratory examination values.

Results: No significant difference in the proportion of infection was noted among various lung lobes $(P>0.05)$. The $\mathrm{POI}$ of total lung was negatively correlated with the peripheral blood lymphocyte percentage (L\%) $(r=-0.633, P<$ $0.001)$ and lymphocyte (LY) count $(r=-0.555, P=0.001)$ but positively correlated with the neutrophil percentage (N\%) $(r=0.565, P=0.001)$. Otherwise, the POI was not significantly correlated with the peripheral blood white blood cell (WBC) count, monocyte percentage (M\%) or haemoglobin (HGB) content. In some patients, as the infection progressed, the L\% and LY count decreased progressively accompanied by a continuous increase in the N\%.

Conclusions: Lung lesions in COVID-19 patients are significantly correlated with the peripheral blood lymphocyte and neutrophil levels, both of which could serve as prognostic indicators that provide warning implications, and contribute to clinical interventions in patients.
\end{abstract}

Keywords: COVID-19, SARS-CoV-2, Artificial intelligence, Deep learning, Lymphocyte, Neutrophil, Infection severity

\footnotetext{
* Correspondence: 6397262@qq.com

${ }^{+}$Xingrui Wang and Qinglin Che contributed equally to this work.

'Department of Radiology, Xi'an No.3 Hospital, The Affiliated Hospital of

Northwest University, Shaanxi Province 710018 Xi'an, China

${ }^{2}$ Xi'an Key Laboratory of Cardiovascular and Cerebrovascular Diseases, Xi'an

No.3 Hospital, the Affiliated Hospital of Northwest University, Northwest

University, Xi'an 710018, Shaanxi Province, China

Full list of author information is available at the end of the article
}

(C) The Author(s). 2021 Open Access This article is licensed under a Creative Commons Attribution 4.0 International License, which permits use, sharing, adaptation, distribution and reproduction in any medium or format, as long as you give appropriate credit to the original author(s) and the source, provide a link to the Creative Commons licence, and indicate if changes were made. The images or other third party material in this article are included in the article's Creative Commons. licence, unless indicated otherwise in a credit line to the material. If material is not included in the article's Creative Commons licence and your intended use is not permitted by statutory regulation or exceeds the permitted use, you will need to obtain permission directly from the copyright holder. To view a copy of this licence, visit http://creativecommons.org/licenses/by/4.0/. The Creative Commons Public Domain Dedication waiver (http://creativecommons.org/publicdomain/zero/1.0/) applies to the data made available in this article, unless otherwise stated in a credit line to the data. 


\section{Background}

In December 2019, several cases of pneumonia of unknown causes were reported in Wuhan, Hubei Province, China [1]. It was confirmed that the pneumonia was coronavirus disease 2019 (COVID-19), which was caused by a new coronavirus. Later, the International Committee on Taxonomy of Viruses (ICTV) officially named the virus severe acute respiratory syndrome coronavirus type 2 (SARS-CoV-2). Studies have shown that SARS-CoV-2, which is a beta genera coronavirus, shares similarity with the viruses that cause SRAS and Middle East respiratory syndrome (MERS); however, SARS-CoV-2 is more severely contagious [2]. SARS-CoV-2 is principally transmitted through respiratory droplets (cough, sneeze) and contact, and people of all ages are generally susceptible. The symptoms after infection mainly include fever, dry cough, and fatigue. Most patients have a comparatively good prognosis, and a few quickly progress to acute respiratory distress syndrome (ARDS), sepsis shock, and multiple organ failure, which heralds a poor prognosis. On March 11, 2020, the WHO announced that COVID19 has caused a global pandemic. As of March 15, 2020, COVID-19 had spread in 135 countries and territories around the world, with more than 330,000 cumulative confirmed cases and more than 14,000 deaths [3].

COVID-19 generally attacks within 14 days after infection, and its diagnosis depends on viral nucleic acid testing, which is susceptible to interference by some factors. Moreover, the sensitivity of nucleic acid testing is relatively lower than that of computer tomography (CT) (71\% vs. 98\%) [4]. $\mathrm{CT}$ is recommended for clinical screening and observation of COVID-19 patients due to its high efficiency and objectivity. However, visual inspection of CT imaging cannot attain a quantitative assessment of the infected area and is incapable of accurately judging the patient's progress. At present, artificial intelligence (AI) technology is becoming increasingly mature. This technology is adept in automatically identifying complex patterns in imaging data, can quantitatively evaluate specific imaging features, and has been widely used in the field of medical imaging [5].

COVID-19 mortality is lower but its morbidity is higher compared with SARS and MERS [6,7]. Current evidence shows that the primary diseased region of COVID-19 patients is the lungs, and normal or decreased peripheral leukocytes, as well as reduced lymphocyte counts, are noted $[8,9]$. Based on the current diagnostic sensitivity of high-resolution CT (HRCT), early changes in the lungs of COVID-19 patients are easily detected. To ensure the accuracy of diagnosis, it is necessary to combine CT results with clinical laboratory testing indicators. However, there is a lack of evidence that indicates the exact relationship between COVID-19 progression and laboratory testing indicators. To further clarify the dynamic changes of relevant clinical laboratory test indicators and their significance in the diagnosis and treatment of COVID-19, this study intends to use AI technology to quantitatively evaluate the extent of pulmonary lesions in COVID-19 patients and, in combination with their respective blood observation indexes, to explore the correlation between the two factors, so as to provide a clinical reference.

\section{Methods \\ Study design}

Chest HRCT images and laboratory examination data of COVID-19 patients were extracted, and lesion areas in the CT images were quantitatively segmented and calculated using the deep learning (DL) system. A crosssectional study was conducted to investigate the correlation between lung infection and clinical laboratory indicators in patients with COVID-19 pneumonia.

\section{Participants}

Thirty-one patients with a diagnosis of COVID-19 were collected from January 21, 2020, to February 4, 2020, in Jingmen No.1 People's Hospital, Hubei Province, China. All patients received a respiratory or blood specimen test via fluorescent reverse-transcription polymerase chain reaction (RT-PCR), and the results were positive for new coronavirus nucleic acids. Furthermore, the viral gene sequence must be highly homologous to the new coronavirus sequence. All participants underwent a HRCT scan and peripheral blood laboratory testing on the same day without other basal diseases that may affect laboratory observation indicators (such as combined bacterial infection and immunosuppression).

In order to comprehensively evaluate the severity of the included patients, the clinical classification of each patient was determined based on his (her) clinical manifestations, radiological and laboratory examination results to serve as a supporting data for the degree of pulmonary infection. The new coronavirus pneumonia diagnosis and treatment plan (trial version 7) (ncpDTP7) developed by the National Health Commission of the People's Republic of China [10] was used as a diagnostic standard. These patients have not been reported in any other submission by anyone else.

\section{Imaging data acquisition and post-processing}

HRCT images were collected in the Department of Radiology, Jingmen No.1 People's Hospital, Hubei Province. A GE MEDICAL SYSTEMS LightSpeed VCT scanner was used. All patients were supine, and the images were captured after the patients were instructed to hold his (her) breath. The following scanning parameters were employed: slice thickness, $1.25 \mathrm{~mm}$; field of view (FOV), $354.0 \mathrm{~mm}$; tube voltage, $120 \mathrm{kV}$; tube current, $278 \mathrm{~mA}$; and image zoom, 1.00. The AI analysis software used for 
image processing was a deep learning system developed by Shanghai United Imaging Intelligence Co., Ltd. and Shanghai Public Health Clinical Center, Fudan University (New Coronavirus Pneumonia Auxiliary Analysis Software, version number: Full-uAI-Discover-NCP.R001.0.0.15980) [11]. The lung window (with window width $1200 \mathrm{HU}$ and window level $-600 \mathrm{HU}$ ) was used for image reading.

\section{Laboratory inspection data collection}

We scrutinized the clinical data of all laboratoryconfirmed COVID-19 patients in the in-hospital medical records system, including clinical charts, laboratory test results, radiological diagnosis opinions and nursing records, and extracted the clinical laboratory examination indicators of each patient through standardized data collection. After the collection of clinical laboratory inspection indicators, the data were independently reviewed and checked by two researchers to ensure that the relevant values were accurate.

\section{Observation indicators}

The original HRCT images of 31 patients were extracted from the Picture Archiving and Communication System (PACS), and all patients were randomly numbered for identification (patient 1 , patient $2, \ldots$, patient 31 ). The lungs of each patient were divided into 20 bronchopulmonary segments based on anatomical division by DL system, and lesions of the whole lung and each lung lobe were calculated. The infection regions were determined through identifying ground-glass opacity (GGO) $(-750$ $\mathrm{HU}$ to $-300 \mathrm{HU})$ and consolidation component $(-300$ $\mathrm{HU}$ to $50 \mathrm{HU}$ ) in the lungs. Besides, a small number of voxels with Hounsfield unit (HU) values not falling within the interval $[-750,50]$ were surrounded by GGOs or consolidation area, which were also designated as the infected area by the system [11]. Specific steps: 1) input the HRCT images into the DL automatic segmentation system; 2) calculate the quantitative metrics that characterize infected regions, including but not limited to the volume of infection (VOI) and the percentage of infection (POI) in the whole lung, lung lobes and bronchopulmonary segments.

Figure 1 shows the software interface obtained by inputting the original HRCT images of one patient into the DL automatic segmentation system. The HU histogram within the infection regions can be visualized. Figure 2 shows the CT image segmentation results of typical COVID-19 infection cases at three different infection stages: the early stage, progressive stage, and severe stage. The contour drawn by the DL system coincided with the lesion boundary visible in the CT image.

The peripheral blood white blood cell (WBC) count, neutrophil percentage (N\%), lymphocyte percentage (L\%), monocyte percentage (M\%), lymphocyte (LY) count, and

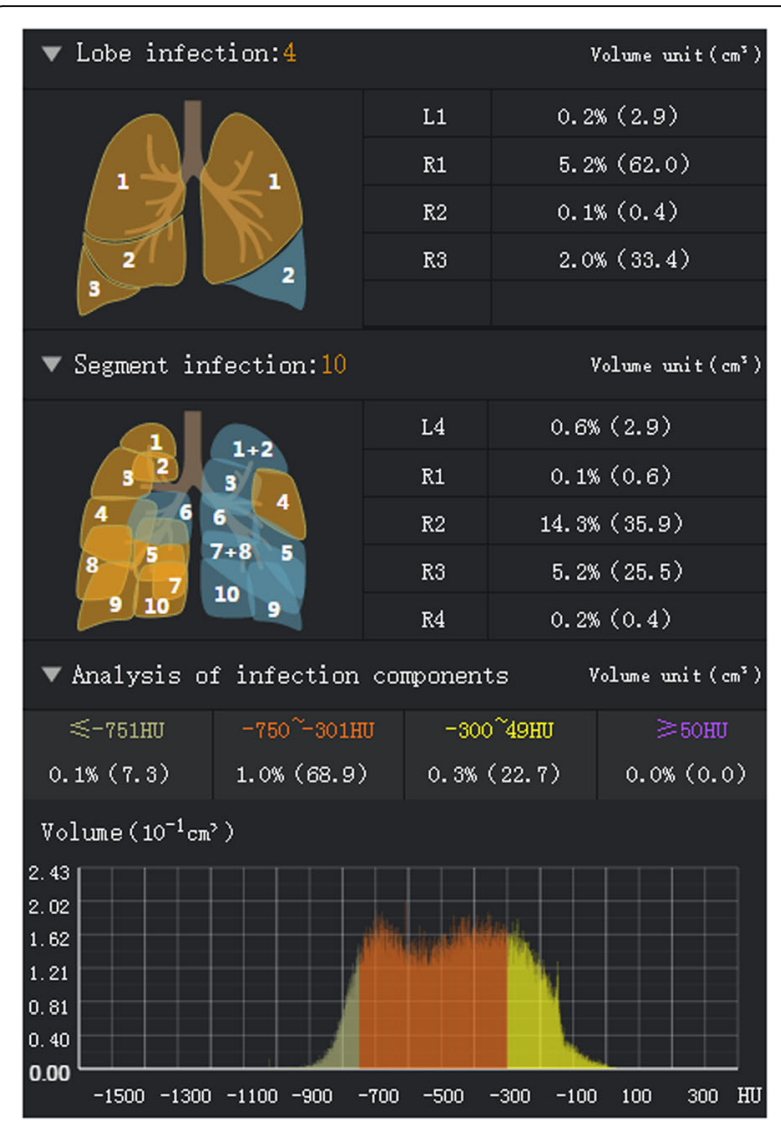

Fig. 1 Software interface obtained by inputting original HRCT images of one patient into the DL system. The VOls and POls in the lung lobes and bronchopulmonary segments are presented; the $\mathrm{HU}$ histogram within the infection regions can be visualized

haemoglobin (HGB) content were extracted. Laboratory examination and HRCT image acquisition of each patient were completed on the same day. Two independent researchers verified all the values.

\section{Statistical analysis}

The Shapiro-Wilk test was used to verify the normality of the data. The Kruskal-Wallis test was performed on the POIs of the following five lung lobes: the left upper lobe, left lower lobe, right upper lobe, right middle lobe, and right lower lobe. The Spearman correlation test was carried out to analyse the correlation between the total pulmonary POI and the peripheral blood WBC count, N\%, L\%, M\%, LY count, and HGB content. SPSS 19.0 statistical software (IBM Company, Armonk, NY) was employed.

\section{Results}

A total of 31 COVID-19 patients were involved in this study, including 18 males (58.1\%) and 13 females (41.9\%). The patients were 17 to 80 years old with an average age of $42.6 \pm 16.0$ years. Of the 31 patients, 8 

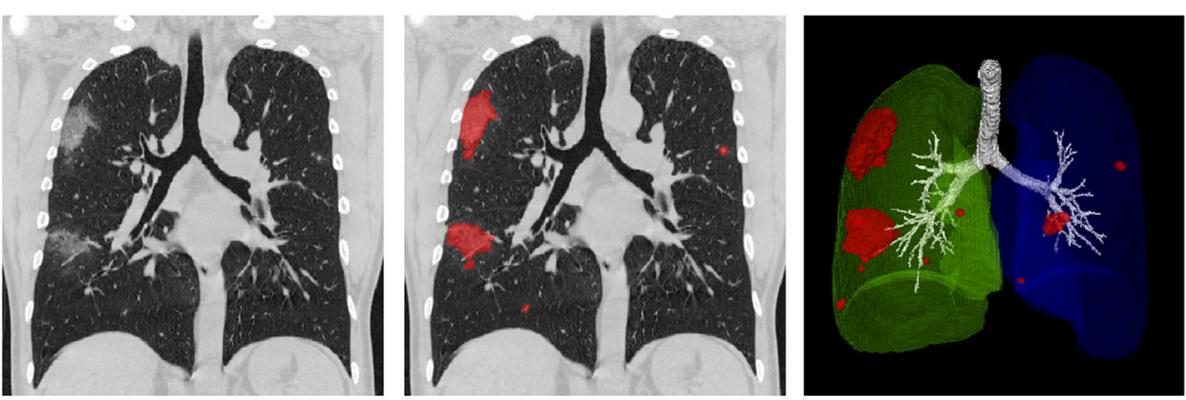

(a) Patient 12 (early stage)
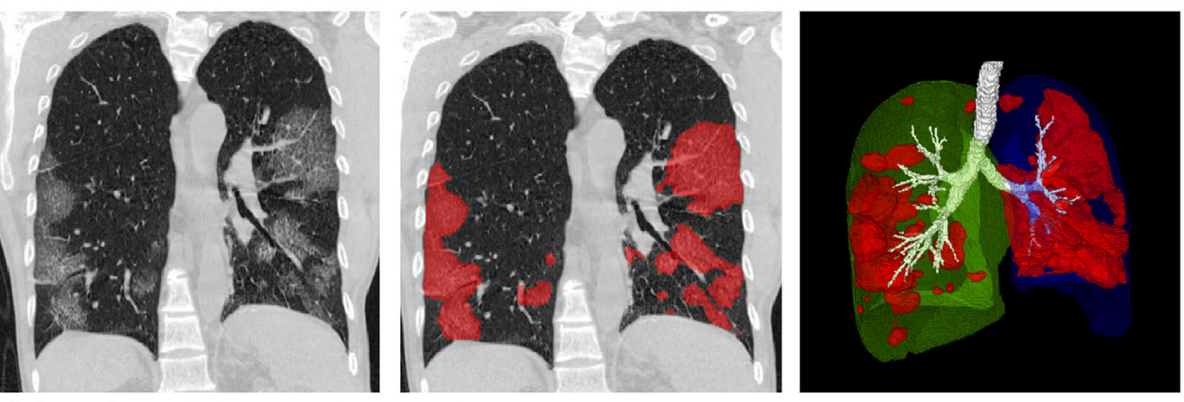

(b) Patient 19 (progressive stage)
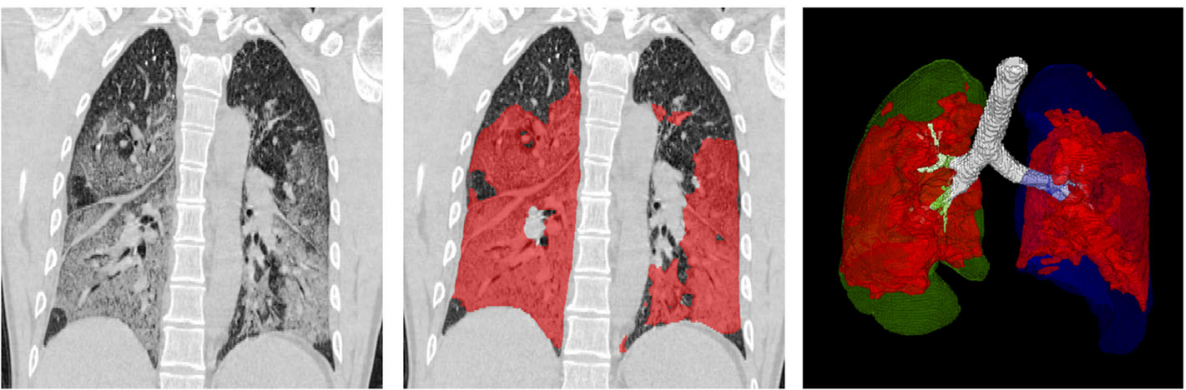

(c) Patient 4 (severe stage)

Fig. 2 Lesion segmentation results of three COVID-19 cases displayed using the software post-processing platform. Rows 1-3: early, progressive, and severe stages. Columns 1-3: CT image, CT images overlaid with segmentation, and 3D surface rendering of segmented infections

(25.8\%) had one or more comorbidities. The most common symptoms at the initial stage of illness were fever [29 (93.5\%)], cough [15 (48.4\%)]. Less common symptoms were fatigue [2 (6.5\%)], myalgia [1 (3.2\%)] and diarrhea $[1(3.2 \%)]$. The average interval from illness onset to CT scan was $8.8 \pm 5.7$ days, and the mean duration of hospitalization before the CT scan was $2.6 \pm 3.5$ days. The average proportion of affected lungs calculated by AI was $8.91 \% \pm 15.01 \%$. Thereinto, 21 patients $(67.7 \%)$ had an infection of less than $5 \%$; 5 patients $(16.1 \%)$ were between 5 and $20 \%$, the other $5(16.1 \%)$ were for more than $20 \%$. According to the criteria in ncpDTP-7, 23 patients $(74.2 \%)$ were moderate, 4 (12.9\%) were severe and remaining $4(12.9 \%)$ met the critical type. More demographic data and laboratory tests of the study group are listed in Table 1.

The status of pulmonary infection is presented in Table 2. No significant difference was noted among the proportions of pulmonary lobe infection $(P>0.05)$. Correlation analysis found that the total pulmonary POI was negatively correlated with the $\mathrm{L} \%(r=-0.633, P<$ 0.001 , Fig. $3 \mathrm{a})$ and the LY count $(r=-0.555, P=0.001$, Fig. 3b) but positively correlated with the $\mathrm{N} \%(r=0.565$, $P=0.001$, Fig. 3c). The peripheral blood WBC count, M\% and HGB content did not significantly correlate with the total POI of the lungs (Table 3 ).

In the data we collected, of note, several patients in different disease periods showed a dynamic trend of progressively decreased L\% and LY count, accompanied by continuously increased N\%, which correlated with the increase in the pulmonary infection volume (Table 4, Fig. 4).

\section{Discussion}

The lungs are more vulnerable to SARS-CoV-2 than other organs. Pathological studies have shown that the 
Table 1 Demographics, clinical characteristics, and laboratory findings of COVID-19 patients

\begin{tabular}{l} 
Characteristics \\
\hline Age, years \\
$<40$ \\
$40-60$ \\
$>60$ \\
Sex \\
Female \\
Male \\
Hypertension/ diabetes/cardiovascular disease/ \\
cerebrovascular disease/COPD/kidney disease \\
Signs and symptoms \\
Fever \\
Cough \\
Fatigue \\
Myalgia \\
Diarrhea
\end{tabular}

Interval from illness onset to CT scan, days

Days of clinical intervention before $C T$ scan

Proportion of affected lungs calculated by $\mathrm{Al}(\%)$

$$
\begin{aligned}
& <5 \% \\
& 5-20 \% \\
& >20 \%
\end{aligned}
$$

Clinical classification according to ncpDTP-7

$$
\text { Mild }
$$

Moderate

Severe

Critical

Arterial blood gas on the day of CT scan

$$
\begin{aligned}
& \mathrm{PaO}_{2}, \mathrm{mmHg} \\
& <60 \\
& \geq 60 \\
& \mathrm{PaCO}_{2}, \mathrm{mmHg} \\
& \leq 50 \\
& >50
\end{aligned}
$$

\begin{tabular}{|c|c|c|}
\hline & Characteristics & \\
\hline $42.6 \pm 16.0$ & & 18.1 \\
\hline $16(51.6 \%)$ & AST, U/L & $35.3 \pm 28.6$ \\
\hline 12 (38.7\%) & $\leq 40$ & $21 / 30$ \\
\hline \multirow[t]{2}{*}{$3(9.7 \%)$} & & $(70.0 \%)$ \\
\hline & $>40$ & $\begin{array}{l}9 / 30 \\
(30.0 \%)\end{array}$ \\
\hline 13 (41.9\%) & $\mathrm{ALT}, \mathrm{U} / \mathrm{L}$ & $26.7 \pm 18.4$ \\
\hline $18(58.1 \%)$ & $\leq 35$ & $25 / 30$ \\
\hline \multirow[t]{2}{*}{$8(25.8 \%)$} & & $(83.3 \%)$ \\
\hline & $>35$ & $\begin{array}{l}5 / 30 \\
(16.7 \%)\end{array}$ \\
\hline $29(93.5 \%)$ & CRP, mg/L & $19.2 \pm 30.1$ \\
\hline 15 (48.4\%) & $\leq 5$ & $10 / 21$ \\
\hline $2(6.5 \%)$ & & $(4 / .6 \%)$ \\
\hline 1 (3.2\%) & $>5$ & $\begin{array}{l}11 / 21 \\
(52.4 \%)\end{array}$ \\
\hline $1(3.2 \%)$ & $\mathrm{CK}, \mathrm{U} / \mathrm{L}$ & $148.3 \pm$ \\
\hline $8.8 \pm 5.7$ & & 271.9 \\
\hline $2.6 \pm 3.5$ & $\leq 200$ & $\begin{array}{l}25 / 28 \\
(89.3 \%)\end{array}$ \\
\hline $\begin{array}{l}8.91 \pm \\
15.01\end{array}$ & $>200$ & $\begin{array}{l}3 / 28 \\
(10.7 \%)\end{array}$ \\
\hline
\end{tabular}

Laboratory findings

White blood cell count, $\times 10^{9} / \mathrm{L}$

$$
\begin{aligned}
& <4 \\
& 4-10 \\
& >10 \\
& \text { Lymphocyte percentage }(\%) \\
& \text { Lymphocyte count, } \times 10^{9} / \mathrm{L} \\
& \text { Neutrophil percentage }(\%) \\
& \text { Monocyte percentage }(\%) \\
& \text { Hemoglobin, g/L }
\end{aligned}
$$

$0(0.0 \%)$
Table 1 Demographics, clinical characteristics, and laboratory findings of COVID-19 patients (Continued)

Continues data are expressed as mean $\pm \mathrm{SD}$. Categorical data are presented as $\mathrm{n}(\%)$ or $\mathrm{n} / \mathrm{N}(\%)$, where $\mathrm{N}$ is the total number of patients with available data Abbreviations: COVID-19 coronavirus disease 2019, COPD chronic obstructive pulmonary disease, CT computer tomography, Al artificial intelligence, ncpDTP-7 new coronavirus pneumonia diagnosis and treatment plan (trial version 7), AST aspartate transaminase, ALT alanine transaminase, CRP Creactive protein, CK creatine kinase

$23(74.2 \%)$

$4(12.9 \%)$

$4(12.9 \%)$

primary changes in the lungs of COVID-19 patients include diffuse alveolar injury, fibrinous protein exudation, and alveolar cell desquamation accompanied by transparent membrane formation and lymphocyte-based inflammatory cell infiltration in the stroma [12]. A hand-
$57.7 \pm 19.8$ $4 / 6(66.7 \%)$ $2 / 6(33.3 \%)$

$42.7 \pm 6.8$

$5 / 6(83.3 \%)$

$1 / 6(16.7 \%)$

$4.80 \pm 1.87$

$13(41.9 \%)$

17 (54.8\%)

1 (3.2\%)

$30.3 \pm 13.3$

$1.35 \pm 0.59$

$57.4 \pm 14.1$

$11.0 \pm 3.7$

$131.4 \pm$ ful of autopsy reports have further demonstrated that COVID-19 is a disease that induces multi-organ and multi-system damage and does not simply affect the lungs [13]. The immune system damage caused by SARS-

Table 2 Lung (lobes) average infection volume and proportion

\begin{tabular}{lll}
\hline Anatomical partition & VOI $\left(\mathbf{c m}^{\mathbf{3}}\right)$ & POI (\%) \\
\hline Whole lung & $281.19 \pm 421.55$ & $8.91 \pm 15.01$ \\
Left lung & $115.45 \pm 191.99$ & $7.81 \pm 13.26$ \\
$\quad$ Upper lobe & $57.02 \pm 108.02$ & $7.72 \pm 14.92$ \\
$\quad$ Lower lobe & $58.44 \pm 93.80$ & $10.31 \pm 20.26$ \\
Right lung & $165.73 \pm 243.46$ & $9.98 \pm 17.14$ \\
$\quad$ Upper lobe & $54.39 \pm 89.82$ & $9.50 \pm 18.50$ \\
Middle lobe & $20.44 \pm 39.93$ & $8.20 \pm 18.54$ \\
Lower lobe & $90.91 \pm 123.99$ & $13.54 \pm 22.82$ \\
\hline
\end{tabular}

Data are expressed as mean \pm SD

Abbreviations: VOI volume of infection, $\mathrm{POI}$ percentage of infection 

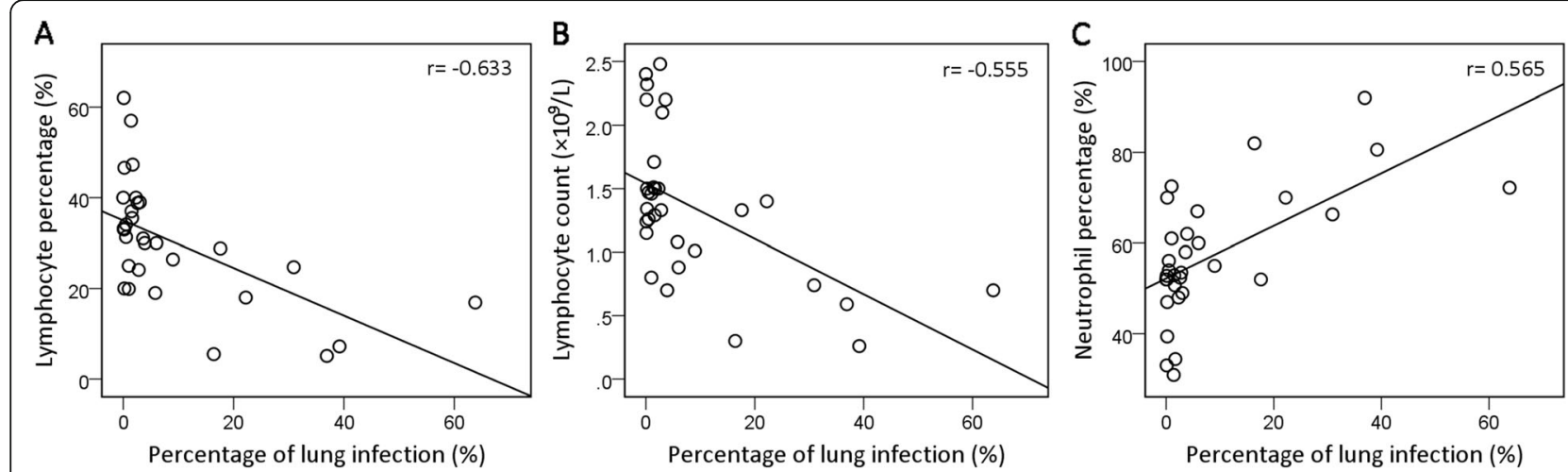

Fig. 3 Correlation between total pulmonary POI and L\%, LY count, or N\%. POI of the total lung was negatively correlated with the $L \%[r=-0.633$, $P<0.001$, (a) $]$ and LY count $[r=-0.555, P=0.001$, (b) $]$ but positively correlated with the $N \%[r=0.565, P=0.001$, (c) $]$

$\mathrm{CoV}-2$ should not be underestimated. The spleen volume of COVID-19 patients is significantly reduced, and the number of $\mathrm{CD}^{+}{ }^{+} \mathrm{T}$ cells and $\mathrm{CD} 8^{+} \mathrm{T}$ cells in the spleen and peripheral lymph nodes is also reduced; such effects are accompanied by tissue degeneration and necrosis as well as a proliferation of macrophages, which are specifically like those noted with SARS-CoV infection [14-16]. Lymphocytopenia seems to potentially signify that COVID19 may deplete and disrupt the immune system in some direct or indirect manner, resulting in an AIDS-like response. Studies on SARS indicate that SARS-CoV cannot infect human lymphocytes and monocytes in vitro and that attacking lymphocytes and mononuclear cells with infectious SARS-CoV, inactivated virus particles, or receptor protein-binding fragments of the virus is unable to trigger an apoptotic response [17]. Besides, autopsies of COVID19 patients yielded negative immunohistochemistry and PCR results from spleen, bone marrow, and peripheral lymphoid tissues. The above results suggest that SARS$\mathrm{CoV}-2$ is unlikely to destroy the human immune system via a direct mechanism. The reason is probably related to the lack of angiotensin-converting enzyme 2 (ACE2) expression in human immune tissues or organs $[18,19]$.

Table 3 Correlation between total lung infection and the clinical laboratory indicators

\begin{tabular}{lll}
\hline & POl of whole lung & \\
\cline { 2 - 3 } & Correlation coefficient $\boldsymbol{(} \boldsymbol{r})$ & $\boldsymbol{P}$-value \\
\hline Lymphocyte percentage & -0.633 & 0.000 \\
Lymphocyte count & -0.555 & 0.001 \\
Neutrophil percentage & 0.565 & 0.001 \\
White blood cell count & 0.057 & $>0.05$ \\
Monocyte percentage & 0.097 & $>0.05$ \\
Haemoglobin content & -0.193 & $>0.05$ \\
\hline
\end{tabular}

*P $<0.05$ was considered statistically significant

Abbreviations: POI percentage of infection
However, the mechanism underlying lymphopenia in peripheral blood of COVID-19 patients remains unclear. There are three possible explanations: a) The inflammatory storm gives rise to the destruction and consumption of lymphocytes. Studies have shown that the strong type I interferon (IFN) response caused by a viral infection and the high levels of glucocorticoids caused by normal stress responses can induce T cell apoptosis [20,21]. Also, the intense cytokine storm itself experienced by SARS patients can induce lymphocyte apoptosis [17, 22], suggesting that lymphocyte apoptosis might exist in COVID-19 patients. b) Reduced lymphocyte production. Any debilitating disease inevitably activates the stress response mediated by the hypothalamicpituitary-adrenal (HPA) axis and increases cortisol secretion. Steroid levels in the blood can significantly affect the number and biological behaviour of lymphocytes in the circulatory system. Robertson et al. reported that glucocorticoids can induce human lymphoblast apoptosis; even under physiological conditions, the number of lymphocytes also has a significant negative correlation with the circadian rhythm of the cortisol content [23-25]. After viral infection, the body exhibits a stress reaction, and the HPA axis is activated to produce more steroids, thus inhibiting the level of lymphocytes in the circulatory system. Of course, the possibility that the lymphocyte levels also change after patients receive an exogenous cortisol treatment cannot be excluded. However, many COVID-19 patients have exhibited a decreasing trend in lymphocytes in peripheral blood before receiving a clinical intervention. At present, controversy exists regarding whether glucocorticoids should be used to relieve the symptoms of patients with severe viral pneumonia [7, 26-28]. The application of glucocorticoids in the treatment of COVID-19 should be considered dialectically. Besides, whether glucocorticoids can cause immunosuppression in SARS-CoV-2-infected patients and the relationship between the dosage of glucocorticoids and the prognosis of patients still require further research. c) Abnormal distribution of lymphocytes in the body. The 
Table 4 Dynamic changes of the lung infection volume and laboratory indicators in some patients

\begin{tabular}{|c|c|c|c|c|}
\hline & Infected lung volume $\left(\mathrm{cm}^{3}\right)$ & Neutrophil percentage (\%) & Lymphocyte percentage (\%) & Lymphocyte count $\left(\times 10^{9} / L\right)$ \\
\hline \multicolumn{5}{|l|}{ Patient 18} \\
\hline Time 1 & 572.2 & 52.0 & 28.8 & 1.33 \\
\hline Time 2 & 957.7 & 72.5 & 19.5 & 1.38 \\
\hline Time 3 & 1596.4 & 95.9 & 1.7 & 0.31 \\
\hline \multicolumn{5}{|l|}{ Patient 22} \\
\hline Time 1 & 202.7 & 57.8 & 28.6 & 0.99 \\
\hline Time 2 & 298.8 & 60.2 & 30.3 & 0.88 \\
\hline \multicolumn{5}{|l|}{ Patient 24} \\
\hline Time 1 & 157.1 & 64.0 & 23.4 & 1.25 \\
\hline Time 2 & 198.3 & 67.2 & 18.9 & 1.08 \\
\hline \multicolumn{5}{|l|}{ Patient 27} \\
\hline Time 1 & 3.4 & 52.0 & 33.3 & 1.15 \\
\hline Time 2 & 330.2 & 70.1 & 19.8 & 0.85 \\
\hline \multicolumn{5}{|l|}{ Patient 28} \\
\hline Time 1 & 852.4 & 66.3 & 24.7 & 0.74 \\
\hline Time 2 & 1299.7 & 85.0 & 8.5 & 0.36 \\
\hline
\end{tabular}

immune response of the respiratory tract to invasive pathogens is initiated by airway epithelial cells. After airway epithelial cell infection, resident respiratory dendritic cells (DCs) are activated by pathogens or antigens to process antigens and simultaneously migrate to peripheral lymphoid organs. After arriving at peripheral lymphoid organs, DCs present the processed antigens to immature $\mathrm{T}$ lymphocytes in the form of the major histocompatibility complex (MHC)-peptide complex. After binding to the MHCpeptide complex, $\mathrm{T}$ cells are activated to proliferate and migrate to the infected site $[29,30]$. This process will inevitably lead to the redistribution of lymphocytes in the lesion and other areas. It is worth noting that the effect of COVID-19 on the immune system does not simply involve reducing the number of lymphocytes via a specific mechanism, which is quite likely attributed to a combination of the above three reasons. The exact mechanism of this change needs to be confirmed by relevant cellular and molecular pathology research. In addition, our study also demonstrated that the number and percentage of lymphocytes decreased progressively as COVID-19 progressed, suggesting that the level of lymphocytes in the blood might be a biomarker to predict the prognosis of COVID-19 patients.

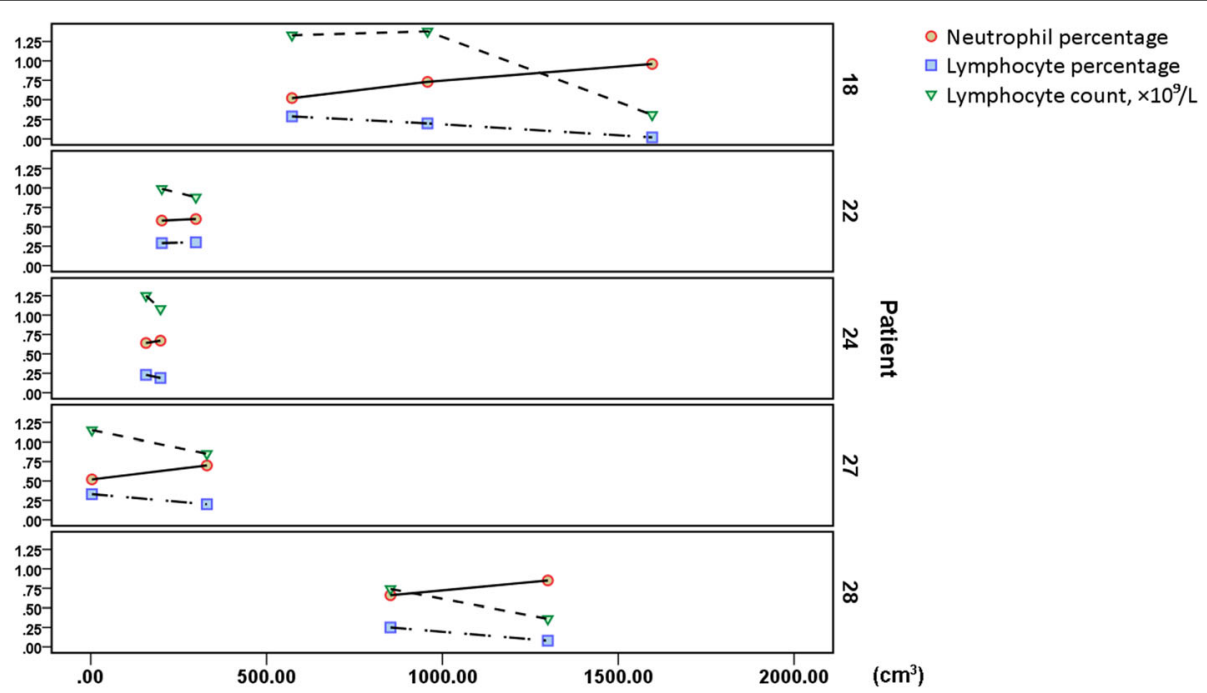

Fig. 4 Dynamic trend of L\%, LY count, and N\% in several patients. As the pulmonary infection volume increased, L\% and LY count exhibit progressively decreased accompanied by continuously increased N\% 
Our study also found a significant positive correlation between the percentage of neutrophils in peripheral blood and the severity of pulmonary infection. Neutrophils are differentiated from hematopoietic stem cells in the bone marrow and exhibit active chemotaxis, phagocytosis, and bactericidal effects. As the most abundant leukocytes in the circulatory system, neutrophils play a central role in the natural immune system and participate in the regulation of adaptive immune responses. Generally, neutrophil activation is more sensitive to bacterial infection, but research on SARS has shown that cytokines and complement activation play an important role in the progression of SARS, which is related to neutrophil activation and aggregation [31,32]. Based on this finding, it is hypothesised that the increased proportion of neutrophils in peripheral blood of patients with COVID-19 may also be related to the production of multiple cytokines (such as IFN- $\gamma$ ) and the activation of the complement system after infection with the virus. Moreover, if the patient is infected with bacteria in the late stage of the disease, the percentage of neutrophils would also increase. A retrospective study involving 1312 patients with SARS reported that the neutrophil count is a highly reliable prognostic indicator of fatality in SARS-CoV-infected patients, predicting relatively high mortality [33]. Evidence also suggests that when people are infected with some severe respiratory viruses (such as SARS-CoV, H5N1), neutrophil infiltration into the lungs will produce high levels of chemokines, such as $\mathrm{C}$ $\mathrm{X}-\mathrm{C}$ motif chemokine 10 (CXCL10), which can induce fulminant pneumonia and aggravate the ARDS [34]. Based on the above reasons, we suggest that the neutrophil level in peripheral blood should be an area of focus during the treatment of COVID-19 patients. Once the neutrophil level in peripheral blood becomes abnormal, certain interventions and related supportive treatment should be administered in time to improve the prognosis and to reduce the fatality rate.

Our research has the following limitations. First, the sample size is small, and the data are not normally distributed. Thus, the information obtained may exhibit deviations. Subsequent research with a larger sample is needed to further reveal the specific relationship between these two factors. Second, the inconsistency in the treatment options of the patients included is also another limitation. Although it has been confirmed that the lymphocyte count in COVID-19 patients is reduced before they receive treatment, it cannot be excluded that different treatment options administered during hospitalization could bias the results.

\section{Conclusions}

In this study, AI was used to assess the extent of pulmonary lesions in COVID-19 patients, and the correlation between overall lung lesions and related clinical laboratory tests was analysed, revealing that the reduction in the peripheral blood lymphocyte level and the increase in the neutrophil level caused by COVID-19 were significantly related to the degree of lung lesions. Moreover, the dynamic changes of the two indicators after SARS-CoV-2 infection could play a role in guiding the choice of treatment options for patients.

\section{Supplementary Information}

The online version contains supplementary material available at https://doi. org/10.1186/s12879-021-05839-9.

Additional file 1. Overal pulmonary infection results in 31 patients with COVID-19 calculated by Al system.

Additional file 2. Clinical laboratory examination results of 31 patients with COVID-19 corresponding to pulmonary VOI and POI at the same stage of the disease.

\section{Abbreviations}

COVID-19: Coronavirus disease 2019; SARS-CoV-2: Severe acute respiratory syndrome coronavirus type 2; MERS: Middle East respiratory syndrome; ARDS: Acute respiratory distress syndrome; WHO: World Health Organization; $C T$ : Computer tomography; Al: Artificial intelligence; DL: Deep learning; RTPCR: Reverse-transcription polymerase chain reaction; HRCT: High-resolution CT; ncpDTP-7: New coronavirus pneumonia diagnosis and treatment plan (trial version 7); PACS: picture archiving and communication system; GGO: Ground-glass opacity; HU: Hounsfield unit; VOI: Volume of infection; POI: Percentage of infection; WBC: White blood cell; N\%: Neutrophil percentage; L\%: Lymphocyte percentage; M\%: Monocyte percentage; LY: Lymphocyte; HGB: Haemoglobin; ACE2: Angiotensin-converting enzyme 2; IFN: Interferon; HPA: Hypothalamic-pituitary-adrenal; DCs: Dendritic cells; MHC: Major histocompatibility complex; CXCL10: C-X-C motif chemokine 10

\section{Acknowledgements}

Not applicable.

\section{Authors' contributions}

XW completed the data integration and statistical analysis and was a major contributor to manuscript writing. QC was responsible for extracting the imaging data and collecting the laboratory examination data. XJ and $\mathrm{HL}$ completed the $C T$ image inspection to ensure the quality of the data. XM, $L Z, R J, W B$ and $L T$ finished the inspection and verification of the laboratory examination results of patients. YG finalized the study design and provided technical support. All the authors read and approved the final manuscript.

Funding

Not applicable.

\section{Availability of data and materials}

The datasets collected and analysed during the current study are included in this published article and its supplementary information files. More detail information on the datasets and materials used in this study are available from the corresponding author on reasonable request.

\section{Ethics approval and consent to participate}

The study protocol was approved by the Clinical Research Ethics Committee of Jingmen No.1 People's Hospital. Due to the infectiousness of the disease and the regulations of nosocomial infection prevention and control, we failed to directly contact with the COVID-19 patients. However, with clinicians' assistance, verbal informed consent was obtained from patients or their family members, and this procedure was approved by the ethics committee. All patients' data were anonymised before use.

Consent for publication

Not applicable. 


\section{Competing interests}

The authors declare that they have no competing interests.

\section{Author details}

'Department of Radiology, Xi'an No.3 Hospital, The Affiliated Hospital of Northwest University, Shaanxi Province 710018 Xi'an, China. ${ }^{2}$ Xi'an Key Laboratory of Cardiovascular and Cerebrovascular Diseases, Xi'an No.3 Hospital, the Affiliated Hospital of Northwest University, Northwest University, Xi'an 710018, Shaanxi Province, China. ${ }^{3}$ Department of Radiology, Jingmen No.1 People's Hospital, Jingmen 448000, Hubei Province, China.

Received: 18 April 2020 Accepted: 25 January 2021

\section{Published online: 18 February 2021}

\section{References}

1. Zhu N, Zhang D, Wang W, Li X, Yang B, Song J, Zhao X, Huang B, Shi W, Lu $\mathrm{R}$, et al. A novel coronavirus from patients with pneumonia in China, 2019 N Engl J Med 2020;382(8):727-733.

2. Chen J. Pathogenicity and transmissibility of 2019-nCoV-A quick overview and comparison with other emerging viruses. Microbes Infect. 2020;22(2):69-71.

3. World Health Organization. Coronavirus disease (COVID-19) pandemic.2020. Available from: https://www.who.int/emergencies/diseases/novelcoronavirus-2019. Accessed 15 Mar 2020.

4. Fang $Y$, Zhang $H$, Xie J, Lin M, Ying L, Pang P, Ji W. Sensitivity of chest $C T$ for COVID-19: comparison to RT-PCR. Radiology. 2020;296(2):E115-E7.

5. Hosny A, Parmar C, Quackenbush J, Schwartz LH, Aerts HJWL. Artificial intelligence in radiology. Nat Rev Cancer. 2018;18(8):500-10.

6. Epidemiology Working Group for NCIP Epidemic Response. Chinese Center for Disease Control and Prevention. The epidemiological characteristics of an outbreak of 2019 novel coronavirus diseases (COVID-19) in China. Zhonghua liu xing bing xue za zhi. 2020;41(2):145-51.

7. Onder G, Rezza G, Case-Fatality Rate BS. Characteristics of patients dying in relation to COVID-19 in Italy. JAMA. 2020;323(18):1775-6.

8. Huang C, Wang Y, Li X, Ren L, Zhao J, Hu Y, Zhang L, Fan G, Xu J, Gu X, et al. Clinical features of patients infected with 2019 novel coronavirus in Wuhan, China. Lancet. 2020:395(10223):497-506.

9. Wang D, Hu B, Hu C, Zhu F, Liu X, Zhang J, Wang B, Xiang H, Cheng Z, Xiong Y, et al. Clinical characteristics of 138 hospitalized patients with 2019 novel coronavirus-infected pneumonia in Wuhan, China. JAMA 2020;323(11): 1061-1069.

10. National Health Commission of the People's Republic of China. Notice on the novel coronavirus infection diagnosis and treatment plan (trial version 7) (in Chinese). In: Medical Letter Issued by General Office of National Health Commission (2020) No.184. Beijing, 2020. Available from: http://www. nhc.gov.cn/yzygj/s7653p/202003/46c9294a7dfe4cef80dc7f5912eb1989.shtml. Accessed 15 Mar 2020.

11. Shan F, Gao Y, Wang J, Shi W, Shi N, Han M, Xue Z, Shen D, Shi Y. Abnormal lung quantification in chest $C T$ images of COVID-19 patients with deep learning and its application to severity prediction. Med Phys. 2020; https://doi.org/10.1002/mp.14609.

12. Xu Z, Shi L, Wang Y, Zhang J, Huang L, Zhang C, Liu S, Zhao P, Liu H, Zhu L, et al. Pathological findings of COVID-19 associated with acute respiratory distress syndrome. Lancet Respir Med. 2020;8(4):420-2.

13. Liu Q, Wang RS, Qu GQ, Wang YY, Liu P, Zhu YZ, Fei G, Ren L, Zhou YW, Liu $\mathrm{L}$. Gross examination report of a COVID-19 death autopsy. Fa yi xue za zhi. 2020;36(1):21-3

14. Yao XH, Li TY, He ZC, Ping YF, Liu HW, Yu SC, Mou HM, Wang LH, Zhang HR, Fu WJ, et al. A pathological report of three COVID-19 cases by minimal invasive autopsies. Zhonghua bing li xue za zhi. 2020;49(5):411-7.

15. Liu J, Zheng X, Tong Q, Li W, Wang B, Sutter K, Trilling M, Lu M, Dittmer U, Yang D. Overlapping and discrete aspects of the pathology and pathogenesis of the emerging human pathogenic coronaviruses SARS-CoV, MERS-CoV, and 2019-nCoV. J Med Virol. 2020;92(5):491-4.

16. Wang HJ, Du SH, Yue X, Review CCX. Prospect of pathological features of Corona virus disease. Fa yi xue za zhi. 2020;36(1):16-20.

17. Chan PK, Chen GG. Mechanisms of lymphocyte loss in SARS coronavirus infection. Hong Kong Med J. 2008;14(Suppl 4):21-6.

18. Letko M, Marzi A, Munster V. Functional assessment of cell entry and receptor usage for SARS-CoV-2 and other lineage B betacoronaviruses. Nat Microbiol. 2020;5(4):562-9.
19. Hamming I, Timens W, Bulthuis ML, Lely AT, Navis G, van Goor H. Tissue distribution of ACE2 protein, the functional receptor for SARS coronavirus. A first step in understanding SARS pathogenesis. J Pathol. 2004:203(2):631-7.

20. Sobek V, Balkow S, Körner H, Simon MM. Antigen-induced cell death of T effector cells in vitro proceeds via the Fas pathway, requires endogenous interferon- $\gamma$ and is independent of perforin and granzymes. Eur J Immunol. 2002;32(9):2490-9.

21. Bahl K, Kim SK, Calcagno C, Ghersi D, Puzone R, Celada F, Selin LK, Welsh RM. IFN-induced attrition of CD8 T cells in the presence or absence of cognate antigen during the early stages of viral infections. J Immunol. 2006; 176(7):4284-95.

22. Chen RF, Chang JC, Yeh WT, Lee CH, Liu JW, Eng HL, Yang KD. Role of vascular cell adhesion molecules and leukocyte apoptosis in the lymphopenia and thrombocytopenia of patients with severe acute respiratory syndrome (SARS). Microbes Infect. 2006;8(1):122-7.

23. Robertson AM, Bird CC, Waddell AW, Currie AR. Morphological aspects of glucocorticoid-induced cell death in human lymphoblastoid cells. J Pathol. 1978;126(3):181-7.

24. Fauci AS, Dale DC, Balow JE. Glucocorticosteroid therapy: mechanisms of action and clinical considerations. Ann Intern Med. 1976;84(3):304-15.

25. Norris DA, Fine R, Weston WL, Spector S. Monocyte cellular function in asthmatic patients on alternate-day steroid therapy. J Allergy Clin Immunol. 1978;61(4):255-60.

26. Russell CD, Millar JE, Baillie JK. Clinical evidence does not support corticosteroid treatment for 2019-nCoV lung injury. Lancet. 2020;395(10223): 473-5.

27. Chen RC, Tang XP, Tan SY, Liang BL, Wan ZY, Fang JQ, Zhong N. Treatment of severe acute respiratory syndrome with glucosteroids: the Guangzhou experience. Chest. 2006;129(6):1441-52.

28. Villar J, Ferrando C, Martínez D, Ambrós A, Muñoz T, Soler JA, Aguilar G, Alba F, González-Higueras E, Conesa LA, et al. Dexamethasone treatment for the acute respiratory distress syndrome: a multicentre, randomised controlled trial. Lancet Respir Med. 2020;8(3):267-76.

29. Belz GT, Smith CM, Kleinert L, Reading P, Brooks A, Shortman K, Carbone FR, heath WR. Distinct migrating and nonmigrating dendritic cell populations are involved in MHC class I-restricted antigen presentation after lung infection with virus. Proc Natl Acad Sci U S A 2004;101(23):8670-8675.

30. Norbury CC, Malide D, Gibbs JS, Bennink JR, Yewdell JW. Visualizing priming of virus-specific CD8+ T cells by infected dendritic cells in vivo. Nat Immunol. 2002;3(3):265-71.

31. Wong CK, Lam CW, Wu AK, Ip WK, Lee NL, Chan IH, Lit LC, Hui DS, Chan $\mathrm{MH}$, Chung SS, et al. Plasma inflammatory cytokines and chemokines in severe acute respiratory syndrome. Clin Exp Immunol. 2004;136(1):95-103.

32. Gralinski LE, Sheahan TP, Morrison TE, Menachery VD, Jensen K, Leist SR, Whitmore A, Heise MT, Baric RS. Complement activation contributes to severe acute respiratory syndrome coronavirus pathogenesis. mBio. 2018;9(5)

33. Chan JC, Tsui EL, Wong VC. Prognostication in severe acute respiratory syndrome: a retrospective time-course analysis of 1312 laboratory-confirmed patients in Hong Kong. Respirology. 2007;12(4):531-42.

34. Ichikawa A, Kuba K, Morita M, Chida S, Tezuka H, Hara H, Sasaki T, Ohteki T, Ranieri VM. dos Santos CC, et al. CXCL10-CXCR3 enhances the development of neutrophil-mediated fulminant lung injury of viral and nonviral origin. Am J Respir Crit Care Med. 2013;187(1):65-77.

\section{Publisher's Note}

Springer Nature remains neutral with regard to jurisdictional claims in published maps and institutional affiliations. 